\title{
Antispastic Effect of Electroacupuncture on Upper Extremity in Stroke Patients by T-reflex Study : A Single-Blind, Randomized Controlled, Preliminary Study
}

\author{
Min Kyoung Cho ${ }^{1}$, In Lee ${ }^{2,4}$, Jung Nam Kwon ${ }^{2,4}$, Byung Cheul Shin ${ }^{3,4}$ \\ Sung Hwa $\mathrm{Ko}^{5}$, Hyun Yoon $\mathrm{Ko}^{6}$, Yong Il Shin ${ }^{6}$, Jin Woo Hong ${ }^{2,4}$ \\ ${ }^{1}$ Department of Internal Medicine of Korean Medicine, \\ Korean Medicine Hospital of Daejeon University, Cheongju, Republic of Korea \\ ${ }^{2}$ Department of Internal Medicine of Korean Medicine, \\ Pusan National University Korean Medicine Hospital, Yangsan, Republic of Korea \\ ${ }^{3}$ Department of Rehabilitation Medicine of Korean Medicine, \\ Pusan National University Korean Medicine Hospital, Yangsan, Republic of Korea \\ ${ }^{4}$ Pusan National University School of Korean Medicine, Yangsan, Republic of Korea \\ ${ }^{5}$ Department of Rehabilitation Medicine, Pusan National University Hospital, Pusan, Republic of Korea \\ ${ }^{6}$ Department of Rehabilitation Medicine, Pusan National University Yangsan Hospital, \\ Pusan National University School of Medicine, Yangsan, Republic of Korea
}

\begin{abstract}
Objectives: There have been several studies evaluated effect of electroacupuncture (EA) on spasticity but most studies could not assess spasticity quantitatively because they used clinical rating scales for assessment spasticity. The objective of this study is to evaluate effect of EA on poststroke spasticity quantitatively using tendon reflex (T-reflex). Methods: 29 stroke patients with upper extremity spasticity were randomized to EA group and control group. The EA group received combined EA and rehabilitation therapy 5 times a week for 3 weeks. Acupuncture treatment was given at Jian Yu (LI 15), Qu Chi (LI 11), Shao Hai (HT 3), Wai Guan (TE 5), He Gu (LI 4), Lie Que (LU 7), Hou Xi (SI 3) of the affected side, 30 minutes of electrical stimulation with a frequency of $40 / 13 \mathrm{~Hz}$ was applied at $\mathrm{Qu}$ Chi (LI 11), He Gu (LI 4). The control group received only rehabilitation therapy. The efficacy of treatment was assessed using T-reflex latency and amplitude, modified Ashworth scale (MAS) of biceps brachii, brachioradialis and triceps brachii. Fugl-Meyer motor function assessment (FMA) and functional independence measure (FIM) were also measured to assess motor function and functional independence. All outcomes were measured before treatment, immediately after 3 weeks of treatment and 1 week after 3 weeks of treatment.

Results: No statistically significant differences were found in outcomes including T-reflex between the study groups except for FIM values immediately after 3 weeks of treatment $(\mathrm{p}=0.037)$.

Conclusions: These results suggest that 3 weeks of EA does not reduce poststroke upper extremity spasticity electrophysiologically and clinically. However, small sample sizes and contradictory tendency between results from T-reflex and those from MAS require cautious judgement on interpretation of the results. A larger, well-designed clinical trials for quantitative evaluation of effect of EA on poststroke spasticity will be needed.
\end{abstract}

Key Words : Poststroke spasticity, Electroacupuncture, T-reflex study

\footnotetext{
- Received : 19 November 2015

- Revised : 23 December 2015

- Accepted : 24 December 2015

- Correspondence to : Jin Woo Hong

Department of Internal Medicine of Korean Medicine, Pusan National University Korean Medicine Hospital. Beomeo-ri, Mulgeum-eup, Yangsan-si, Gyeongsangnam-do, Korea(Republic of)

Tel : +82-55-360-5962, Fax : +82-55-360-5509, E-mail : jwhong@pusan.ac.kr

- Correspondence to : Yong II Shin

Department of Rehabilitation Medicine, Pusan National University Yangsan Hospital.

Beomeo-ri, Mulgeum-eup, Yangsan-si, Gyeongsangnam-do, Korea(Republic of)

Tel : +82-55-360-2872, Fax : +82-55-360-1524, E-mail : rmshin01@gmail.com
} 


\section{Introduction}

According to Statistics Korea, stroke was the third leading cause of mortality in South Korea in $2012^{1}$. Worldwide stroke-related mortality went up $26 \%$ compared with 1990 and reached 5.9 million in $2010^{2)}$.

Spasticity is one of the most common complications of stroke, estimated prevalence of poststroke spasticity were variable, ranging from $17 \%$ to $42.6 \%$ in the chronic phase ${ }^{3)}$, with the prevalence of disabling spasticity $13 \%{ }^{4)}$.

When severe, spasticity can limit patient's range of motion, functional or gait ability and prolonged spasticity may result in shortening of tendons, soft tissue contracture, pain from immobile joints, as a result, it has bad influence on rehabilitation of stroke patient. Furthermore, spasticity increases economic burden of stroke patient. Lundström et al. reported that direct costs for patients with spasticity were 4 times higher than that for patients without spasticity during the first year after stroke ${ }^{5)}$. Therefore, managing spasticity is very important to improve functional movement and quality of life of stroke patients.

Electroacupuncture (EA) is one of acupuncture modalities administers pulsed electrical stimulation through the skin of the human body and it shows synergic effect of acupoint stimulation and electrical stimulation $^{6}$. EA can concentrate electrical stimulation on any part of the body, regulate stimulus quantity objectively, and lessen systemic side effects because of focal stimulation ${ }^{7)}$.

There have been several studies evaluated effect of EA on poststroke spasticity but most studies could not assess spasticity quantitatively because they mainly used clinical rating scales such as Modified Ashworth scale (MAS) for assessment spasticity $^{8-11)}$. This study was designed to evaluate antispastic effect of EA on poststroke spasticity quantitatively using tendon reflex (T-reflex).

T-reflex is an electrophysiologic test quantifying stretch reflex, that is muscle contraction in response to stretching by tendon tap ${ }^{12)}$, and assessing spasticity sensitively ${ }^{13)}$. This report presents the results of the study, which compared the effect of combined EA and rehabilitation therapy with that of rehabilitation -alone therapy on poststroke upper extremity spasticity using T-reflex quantitatively.

\section{Subjects and Methods}

\section{Subjects}

Patients who were 18 years or older and hospitalized in department of rehabilitation medicine, Pusan National University Yangsan Hospital during the period from November 2010 to March 2011 were recruited. Among them, patients who were diagnosed with stroke by CT or MRI of the brain, over 2 weeks out from the onset of the stroke and had 2 or more muscles of biceps brachii, brachioradialis, triceps brachii that were assessed grade 1 or more by MAS of the affected side were enrolled for this study. Patients were excluded if they had (a) participated in other clinical study within 30 days before this study (b) severe neurological or psychological disorder except for stroke (c) past history of hypersensitivity to EA (d) difficulty in being treated with acupuncture because of edema, skin diseases, etc. (e) been thought to be inadequate for this study. All subjects were randomized to EA group and control group by a Physiatrist using SPSS random number generation. For all subjects, conventional treatment for stroke management was allowed but dosage of antispastic drugs was fixed and new antispastic drugs was not allowed during this study.

\section{Methods}

A parallel design clinical trial was conducted for this study. All subjects continued to receive previous rehabilitation therapy once daily, 5 times a week 
according to their own neurological status and functional recovery during this study.

The EA group received additional EA once daily, 5 times a week. Acupuncture treatment was given at acupoint Jian Yu (LI 15), Qu Chi (LI 11), Shao Hai (HT 3), Wai Guan (TE 5), He Gu (LI 4), Lie Que (LU 7), Hou Xi (SI 3) of the affected side using disposable stainless steel needles (diameter $0.25 \mathrm{~mm}$, length $4 \mathrm{~cm}$, Dongbang Acupuncture Inc., Korea). The depth of needle insertion was different according to acupoint but it was about 10 20 mm. Thereafter, 30 minutes of electrical stimulation (ES-160, ITO co., LTD., Japan) with a frequency of 40/13 Hz was applied at Qu Chi (LI 11), He Gu (LI 4). The intensity of electrical stimulation was adjusted to be strong enough to elicit visible muscle contractions but not painful for subjects. EA was given by traditional Korean medicine clinician with more than 4 years of career. The study protocol was registered at the Clinical Research Information Service (CRIS) which is an online registration system for clinical trials in South Korea (registration number: KCT0001422, https://cris.nih.go.kr/cris/search/ search_result_st01_en.jsp?seq=4834\&type=).

\section{Assessments}

1) T-reflex (Tendon reflex)

T-reflex, which is an electrophysiologic test quantifying stretch reflex was used to assess spasticity quantitatively. T-reflex was measured before treatment, immediately after 3 weeks of treatment and 1 week after 3 weeks of treatment in all subjects. To measure T-reflex, electromyogram Dantec Keypoint (Medtronic Functional Diagnostics, Skovlunde, Denmark) with a speed of $10 \mathrm{msec} /$ division, a sensitivity of $0.2 \sim 2$ $\mathrm{mV} /$ division, a frequency range of $10 \mathrm{~Hz} \sim 10 \mathrm{kHz}$ was used. The test muscles were biceps brachii, brachioradialis and triceps brachii of the affected side. At the middle of the each muscle, an activated electrode was attached, and an indifferent electrode to estimate was attached to $5 \mathrm{~cm}$ below of the activated electrode. Then the tendon area was tapped by an electric reflex hammer to elicit tendon reflex, and latency and amplitude from the recorded waveform was measured. Latency is elapsed time between the tendon tap stimulus and the first deflection from the recorded waveform, amplitude is the difference between positive and negative peaks in terms of $\mathrm{mV}^{14)}$. Latencies of three muscles were added together and indicated Total Latency Values, amplitudes of three muscles were added together and indicated Total Amplitude Values.

\section{2) MAS (Modified Ashworth scale)}

MAS was used to assess spasticity clinically (Table 1.). MAS was measured before treatment, immediately after 3 weeks of treatment and 1 week after 3 weeks of treatment in all subjects. The test muscles were biceps brachii, brachioradialis and triceps brachii of the affected side. The subject was relaxed with upper extremity of the affected side fixed not to move. Then the rater measured resistance of the muscle while extending each muscle with medium speed and repeated measurement to reduce intra-rater error. For statistical analysis, the values of MAS were changed as follows; Grade 1=1, Grade $1+=2$, Grade $2=3$, Grade 3=4, Grade $4=5$. The changed values were added together and indicated Total MAS Values.

3) FMA (Fugl-Meyer motor function assessment), FIM (Functional independence measure)

To assess motor function of upper extremity and functional independence, Fugl-Meyer motor function assessment (FMA) and functional independence measure (FIM) were measured before treatment, immediately after 3 weeks of treatment and 1 week after 3 weeks of treatment in all subjects.

Each item on the FMA is rated on a 3 point ordinal scale and scored as follows; cannot perform $=0$ point, performs partially $=1$ point, performs fully $=2$ 
points. FMA assesses motor function, sensation, balance, joint range of motion, pain and maximum score is 226 points but only motor function (66 points) and sensation (12 points) of upper extremity were assessed in this study.

FIM is an evaluative instrument for activities of daily living and consists of 18 items, grouped into motor (13 items) and cognition (5 items). Scores of each item on the FIM ranges from 1 point to 7 points according to performance and maximum score is 126 points (Table 1.).

Table 1. MAS (Modified Ashworth scale)

\begin{tabular}{|c|c|}
\hline Grade & Description \\
\hline 0 & No increase in muscle tone. \\
\hline 1 & $\begin{array}{l}\text { Slight increase in muscle tone, manifested by a catch } \\
\text { and release or by minimal resistance at the end of the } \\
\text { ROM when the affected part(s) is moved in flexion or } \\
\text { extension. }\end{array}$ \\
\hline $1+$ & $\begin{array}{l}\text { Slight increase in muscle tone, manifested by a catch, } \\
\text { followed by minimal resistance throughout the } \\
\text { remainder (less than half) of the ROM. }\end{array}$ \\
\hline 2 & $\begin{array}{l}\text { More marked increase in muscle tone through most of } \\
\text { the ROM, but affected part(s) easily moved. }\end{array}$ \\
\hline 3 & $\begin{array}{l}\text { Considerable increase in muscle tone, passive } \\
\text { movement difficult. }\end{array}$ \\
\hline 4 & Affected part(s) rigid in flexion or extension. \\
\hline
\end{tabular}

\section{Statistical analysis}

All statistical analyses were performed using PASW Statistics 18.0 in this study. To compare baseline characteristics between the study groups, according to normality, we used the Chi-square test or Fisher's exact test for categorical data and Independent t-test or Mann-Whitney U-test for continuous data.

To analyse efficacy of treatment within each group, we compared all outcomes measured before treatment with immediately after 3 weeks of treatment and before treatment with 1 week after 3 weeks of treatment using Paired t-test or Wilcoxon signed rank test according to normality. To analyse efficacy of treatment between the study groups, we compared all outcomes measured before treatment, immediately after 3 weeks of treatment and 1 week after 3 weeks of treatment of both groups using Independent t-test.

All results were shown as frequencies and percentages for continuous data, as mean $\pm \mathrm{SD}$ for categorical data. A P-value of $<0.05$ was considered statistically significant.

\section{Results}

\section{Baseline characteristics of the study groups}

A total of 35 patients were enrolled for this study but 29 completed the whole study course. 3 were excluded because they did not meet inclusion criteria and 3 did not complete the study because they were discharged from the hospital during this study. 29 subjects were randomized to EA group $(n=19)$ and control group $(\mathrm{n}=10)$. Different number of allocated subjects in the study groups was result of high withdrawal rate of control group.

The EA group consisted of 7 (36.8\%) male and $12(63.2 \%)$ female subjects, the control group of 7 (70\%) male and $3(30 \%)$ female subjects. The mean age of the EA group was $61.11 \pm 12.83$ years, the control group was $55.80 \pm 20.27$ years.

The EA group consisted of 8 (42.1\%) ischemic and $11(57.9 \%)$ hemorrhagic stroke, the control group of $6(60 \%)$ ischemic and $4(40 \%)$ hemorrhagic stroke. $8(42.1 \%)$ were affected left side and 11 $(57.9 \%)$ right side in the EA group, percentages of affected side were same in the control group. There were no statistically significant differences between the study groups in all variables (Table 2.). When we were planning this study, we did not placed a limit on time since stroke except over 2 weeks out from the onset of the stroke to raise rate of participation. So it was not available to collect each time since stroke information of subject. No significant side effects were found during the study period in both groups. 


\section{Changes in T-reflex according to treatment period}

1) Changes in Total Latency Values

There were no statistically significant differences within each group and between the study groups (Table 3.).
2) Changes in Total Amplitude Values

There were no statistically significant differences within each group and between the study groups (Table 4.).

\section{Changes in MAS according to treatment period}

Table 2. Baseline Characteristics of the Study Groups

\begin{tabular}{|c|c|c|c|c|}
\hline & & $\begin{array}{l}\text { EA Group } \\
(\mathrm{n}=19)\end{array}$ & $\begin{array}{l}\text { Control Group } \\
(\mathrm{n}=10)\end{array}$ & P-value* \\
\hline \multirow[t]{2}{*}{ Gender, n(\%) } & Male & $7(36.8)$ & $7(70)$ & 0.128 \\
\hline & Female & $12(63.2)$ & $3(30)$ & \\
\hline Age, year(mean \pm SD) & & $61.11 \pm 12.83$ & $55.80 \pm 20.27$ & 0.945 \\
\hline \multirow[t]{2}{*}{ Stroke type, n(\%) } & Ischemia & $8(42.1)$ & $6(60)$ & 0.450 \\
\hline & Hemorrhage & $11(57.9)$ & $4(40)$ & \\
\hline \multirow[t]{2}{*}{ Affected side, n(\%) } & Left & $8(42.1)$ & $5(50)$ & 0.714 \\
\hline & Right & $11(57.9)$ & $5(50)$ & \\
\hline \multirow[t]{2}{*}{ Medical history, $\mathrm{n}(\%)$} & Hypertension & $11(57.9)$ & $5(50)$ & 0.714 \\
\hline & $\mathrm{DM}$ & $6(31.6)$ & $2(20)$ & 0.675 \\
\hline
\end{tabular}

*: Mann-Whitney U-test for age. Fisher's exact test for others.

EA, electroacupuncture; SD, standard deviation; DM, diabetes mellitus

Table 3. Changes in Total T-reflex Latency Values According to Treatment Period

\begin{tabular}{ccc}
\hline Group & Before Treatment & After 3 weeks of Treatment \\
\cline { 2 - 2 } EA Group & $48.10 \pm 6.66$ & Immediately \\
P-value* (Pre-Post) & $46.89 \pm 3.66$ \\
Control Group & $49.77 \pm 8.64$ & 0.448 \\
P-value* (Pre-Post) & $52.24 \pm 8.19$ \\
P-value $\dagger$ (Intergroup) & 0.569 & 0.310 \\
\hline *: Paired t-test $\dagger:$ Independent t-test & 0.076 \\
Total T-reflex Latency Values (ms) are sum of T-reflex latencies in biceps brachii, brachioradialis and triceps brachii of the affected side. \\
Each Pre-Post P-value in both groups is result from comparison with baseline(Before Treatment) value. \\
T-reflex, tendon reflex; EA, electroacupuncture
\end{tabular}

Table 4. Changes in Total T-reflex Amplitude Values According to Treatment Period

\begin{tabular}{|c|c|c|c|}
\hline \multirow{2}{*}{ Group } & \multirow{2}{*}{ Before Treatment } & \multicolumn{2}{|c|}{ After 3 weeks of Treatment } \\
\hline & & Immediately & 1 week \\
\hline EA Group & $5.86 \pm 2.84$ & $6.91 \pm 4.41$ & $7.37 \pm 6.25$ \\
\hline P-value* (Pre-Post) & & 0.208 & 0.445 \\
\hline Control Group & $5.04 \pm 2.19$ & $5.42 \pm 2.47$ & $5.80 \pm 3.05$ \\
\hline P-value* (Pre-Post) & & 0.632 & 0.387 \\
\hline P-value $\dagger$ (Intergroup) & 0.433 & 0.332 & 0.460 \\
\hline
\end{tabular}


No statistically significant differences were found between the study groups, but in the EA group, Total MAS Values measured immediately after 3 weeks of treatment were statistically borderline less than those before treatment $(\mathrm{p}=0.054)$ (Table 5.).

\section{Changes in FMA and FIM according to treatment period}

1) Changes in FMA Values

No statistically significant differences were found between the study groups, but in both groups, FMA Values measured immediately after 3 weeks of treatment and 1 week after 3 weeks of treatment were statistically significant higher than those before treatment (Table 6.).

\section{2) Changes in FIM Values}

In FIM Values measured immediately after 3 weeks of treatment, the EA group was statistically significant higher than the control group $(\mathrm{p}=0.037)$, but there were no statistically significant differences between the study groups in FIM Values measured 1 week after 3 weeks of treatment $(p=0.090)$. In both groups, FIM Values measured immediately after 3 weeks of treatment and 1 week after 3 weeks of treatment were statistically significant higher than those before treatment (Table 7.).

\section{Discussion}

MAS is the most widely used rating scale for assessment spasticity in the clinical environment. MAS is easy and speedy to use, but it cannot reflect slight changes of spasticity because of big gap between grades $^{15)}$, and it is not very reliable for assessment spasticity ${ }^{16}$. To overcome these weak points of MAS, there have been several attempts to assess spasticity quantitatively. Levin and Hui-Chan,

Table 5. Changes in Total MAS Values According to Treatment Period

\begin{tabular}{|c|c|c|c|}
\hline \multirow{2}{*}{ Group } & \multirow{2}{*}{ Before Treatment } & \multicolumn{2}{|c|}{ After 3 weeks of Treatment } \\
\hline & & Immediately & 1 week \\
\hline EA Group & $4.84 \pm 0.96$ & $4.26 \pm 1.20$ & $4.21 \pm 1.48$ \\
\hline P-value* (Pre-Post) & & 0.054 & 0.124 \\
\hline Control Group & $4.80 \pm 0.92$ & $5.10 \pm 0.99$ & $5.20 \pm 1.55$ \\
\hline P-value* (Pre-Post) & & 0.180 & 0.269 \\
\hline $\mathrm{P}$-value $\dagger$ (Intergroup) & 0.910 & 0.069 & 0.103 \\
\hline \multicolumn{4}{|c|}{$\begin{array}{l}\text { *: Wilcoxon signed rank test for immediately after } 3 \text { weeks of treatment in both groups. Paired } t \text {-test for others. } \dagger: \text { Independent } t-\text { test } \\
\text { Total MAS Values are sum of values of MAS in biceps brachii, brachioradialis and triceps brachii of the affected side. } \\
\text { Each Pre-Post P-value in both groups is result from comparison with baseline(Before Treatment) value. } \\
\text { MAS, modified Ashworth scale; EA, electroacupuncture }\end{array}$} \\
\hline
\end{tabular}

Table 6. Changes in FMA Values According to Treatment Period

\begin{tabular}{|c|c|c|c|}
\hline \multirow{2}{*}{ Group } & \multirow{2}{*}{ Before Treatment } & \multicolumn{2}{|c|}{ After 3 weeks of Treatment } \\
\hline & & Immediately & 1 week \\
\hline EA Group & $28.05 \pm 17.29$ & $41.53 \pm 23.85$ & $41.11 \pm 21.08$ \\
\hline P-value* (Pre-Post) & & 0.000 & 0.000 \\
\hline Control Group & $31.30 \pm 20.60$ & $41.00 \pm 28.45$ & $42.60 \pm 29.22$ \\
\hline P-value* (Pre-Post) & & 0.012 & 0.012 \\
\hline $\mathrm{P}$-value $\dagger$ (Intergroup) & 0.656 & 0.958 & 0.875 \\
\hline
\end{tabular}

*: Wilcoxon signed rank test $\dagger$ : Independent t-test

Each Pre-Post $\mathrm{P}$-value in both groups is result from comparison with baseline(Before Treatment) value.

FMA, Fugl-Meyer motor function assessment; EA, electroacupuncture 
Table 7. Changes in FIM Values According to Treatment Period

\begin{tabular}{|c|c|c|c|}
\hline \multirow{2}{*}{ Group } & \multirow{2}{*}{ Before Treatment } & \multicolumn{2}{|c|}{ After 3 weeks of Treatment } \\
\hline & & Immediately & 1 week \\
\hline EA Group & $66.89 \pm 25.89$ & $79.42 \pm 26.02$ & $76.63 \pm 24.37$ \\
\hline P-value* (Pre-Post) & & 0.001 & 0.045 \\
\hline Control Group & $51.70 \pm 33.23$ & $54.40 \pm 34.65$ & $57.00 \pm 35.46$ \\
\hline P-value* (Pre-Post) & & 0.010 & 0.014 \\
\hline P-value $\dagger$ (Intergroup) & 0.184 & 0.037 & 0.090 \\
\hline
\end{tabular}

Goulet et al., Aydin et al., van der Salm et al. and Lee et al. used H-reflex for assessment spasticity ${ }^{7}$ 17-20). Electromyography (EMG) tends to be used for assessment spasticity, but until now, there has not been a study that used T-reflex for evaluation antispastic effect of EA.

T-reflex tests compound muscle action potential, which is occurred when tendon tap stretches a muscle spindle, alpha motor neurons connected with the Ia afferents in the same spinal segment are activated and muscle innervated by the motor neurons is contracted $^{21)}$. T-reflex is easy to test and less painful to examinees $^{22}$. Moreover, T-reflex provides comprehensive informations about spasticity because it activates several neurons such as II afferents and gamma motor neurons, whereas H-reflex activates only Ia afferents $^{23}$. The major parameters of the T-reflex are latency and amplitude ${ }^{14)}$. Latency indicates excitability of alpha motor neurons, so when spasticity occurred, latency decreases because excitability of alpha motor neurons increases ${ }^{13)}$. Amplitude indicates numbers of activated motor neurons by tendon tap ${ }^{23)}$, so when spasticity occurred, amplitude increases because excitability of motor neurons increases.

In this study, we assessed upper extremity spasticity because upper extremity is required more complicated movements than lower extremity. Stroke usually increases tone of antigravity muscles such as flexors of upper extremity and extensors of lower extremity. Because the majority of upper extremity spasticity shows pattern resulted from internal rotation, adduction of the shoulder joint and flexion of the elbow joint ${ }^{24)}$, we assessed spasticity of biceps brachii, brachioradialis, which are involved in elbow flexion and triceps brachii, which is involved in shoulder joint adduction.

In T-reflex latency and amplitude measured during the study period, there were no statistically significant differences within each group and between both groups. As reviewed above, when spasticity occurred, latency decreases and amplitude increases. When spasticity reduced, latency increases and amplitude decreases. In this study, however, although there were no statistical differences, Total Latency Values measured immediately after 3 weeks of treatment decreased in the EA group and increased in the control group compared with those before treatment. That is, EA rather showed a tendency to reduce latency during the treatment period. Meanwhile, EA had no influence on amplitude, but Total Amplitude Values showed a tendency to increase until 1 week after 3 weeks of treatment in both groups.

In MAS measured during the study period, there were also no statistically significant differences within each group and between both groups. But only Total MAS Values measured immediately after 3 weeks of treatment in the EA group were statistically borderline less than those before treatment $(\mathrm{p}=0.054)$. And Total MAS Values showed a tendency to increase in the EA group and decrease in the control 
group until 1 week after 3 weeks of treatment, these results are similar to those of Moon et al., Mukherjee et al. and Wayne et al. who reported that EA group showed a significant reduction of MAS compared with control group ${ }^{8,25,26)}$.

As a result, in this study, results from T-reflex tended to be contradictory to those from MAS, this result is conflicting to that of Min et al. who demonstrated significant correlation between T-reflex and MAS of biceps brachii ${ }^{15)}$. However, Lee et al. and Goulet et al. reported that electrophysiological and clinical rating scales for assessment spasticity may not show similar tendency ${ }^{18,19)}$, therefore, cautious judgement is required to interpret results above.

In this study, FIM Values measured immediately after 3 weeks of treatment were the only outcome that showed statistically significant differences between both groups, the EA group was statistically significant higher than the control group $(\mathrm{p}=0.037)$. However, FMA Values did not show statistically significant differences between both groups during the study period, this means that EA improved functional independence although it could not improve motor function of upper extremity. In this study, whole items of FIM were evaluated, whereas only upper extremity-related items of FMA were evaluated. Because FIM includes items such as self-care, transfers and social cognition, which require lower extremity function or cognitive ability, it is thought that EA played a role in additional improvement of other function beside motor function of upper extremity. Although there were no statistical differences between both groups, FMA Values measured immediately after 3 weeks of treatment and 1 week after 3 weeks of treatment were statistically significant higher than those before treatment in both groups, this may have resulted from effect of rehabilitation therapy, which was given to both groups during the treatment period.

In this study, we carried out follow-up assessment of spasticty in 1 week after 3 weeks of treatment, but
FIM Values measured immediately after 3 weeks of treatment in the EA group, which were statistically significant higher than those in the control group, did not maintain statistical highness in 1 week after 3 weeks of treatment. This means that improvement of functional independence by repetitive EA did not last 1 week.

Overall, 3 weeks of EA could not reduce poststroke spasticity electrophysiologically and clinically, this result is different from that of previous studies, which reported significant antispastic effect of EA. Most of all, T-reflex showed rather aggravated spasticity in the EA group, this may have been caused by differences of electrical stimulation methods.

Electrical stimulation methods for reducing spasticity are roughly classified into applying electrical stimulation to the spastic muscle, to the antagonist of the spastic muscle and to the dermatome of the spastic muscle ${ }^{27}$. Levine et al. explained that when electrical stimulation is applied to the antagonist of a spastic muscle, Ia afferents connected with the antagonist are activated, action potential from the Ia afferents is conducted to the spinal segment, association neurons inhibit motor neurons of the spastic muscle, so spasticity of the muscle is reduced ${ }^{28)}$. Likewise, in a study that aimed to compare antispastic effect according to electrical stimulation methods in patients with spinal cord injury, van der Salm et al. reported that applying electrical stimulation to the spastic muscle reduced muscle stiffness rather than spastic hypertonia because it changed only MAS, not EMG, whereas applying electrical stimulation to the antagonist of the spastic muscle effectively reduced spastic hypertonia because it significantly reduced reflex -initiating angle, which is one of parameters of EMG and regulated by gamma motor neurons ${ }^{7)}$. Qu Chi is one of acupoints administered by electrical stimulation in this study and it is located in brachioradialis, which is an agonist in elbow flexion. Thus, EA at Qu Chi may have limited antispastic effect of EA because electrical stimulation was 
applied to the spastic muscle directly.

Frequency of EA may also have affected results of the study. Electrical stimulation is divided into low frequency $(<10 \mathrm{~Hz})$, middle frequency $(10-100 \mathrm{~Hz})$ and high frequency $(>100 \mathrm{~Hz})$ according to frequency range ${ }^{29)}$. Both low and high frequency electrical stimulation are known to reduce spasticity through increasing release of inhibitory neurotransmitters such as opioid peptides, low frequency electrical stimulation is mediated by $\mu$ or $\delta$-opioid receptors, while high frequency electrical stimulation is mediated by $\kappa$ -opioid receptors ${ }^{30}$. Several frequencies of electrical stimulation with antispastic effect have been reported. In studies on transcutaneous electrical nerve stimulation (TENS), antispastic effect of high frequency TENS such as $100 \mathrm{~Hz}$ have been reported ${ }^{19,31-34)}$, whereas in studies on EA, from $2 \mathrm{~Hz}^{6,26,35)}, 50 \mathrm{~Hz}^{8,10,11,36)}$, to 60 $\mathrm{Hz}^{20,37)}$, antispastic effect of low and middle frequency EA have been reported. Thus until now, the most effective frequency of electrical stimulation for reducing spasticity has not been established. Middle frequency electrical stimulation is partially involved in both low and high frequency electrical stimulation's components ${ }^{38)}, 40 / 13 \mathrm{~Hz}$ middle frequency mixed mode was used for EA in this study. There have been some studies comparing antispastic effect of electrical stimulation according to frequency. Wang et al. reported that antispastic effect of $100 \mathrm{~Hz}$ or $50 \mathrm{~Hz}$ EA was superior to that of $2 \mathrm{~Hz}$ and 100 $\mathrm{Hz}$ was optimal ${ }^{39)}$, Han et al. reported that $100 \mathrm{~Hz}$ TENS was effective in reducing spasticity, but $2 \mathrm{~Hz}$ was not $^{40)}$. As above, high frequency electrical stimulation was reported as being more effective in reducing spasticity than low frequency electrical stimulation, but there have been few studies on antispastic effect of high frequency EA. Therefore, it is thought that further studies on antispastic effect of high frequency EA will be needed.

The period that repetitive EA had showed significant antispastic effect was different from 2 weeks $^{8,20)}, 4$ weeks $^{11)}, 6$ weeks $^{26,35,36)}$, to 10 weeks ${ }^{9)}$ according to studies. The treatment period in this study was 3 weeks, which were relatively shorter than previous studies, so cumulative effect of EA may have been limited in the results. In addition, although there were no statistically significant differences between the study groups in baseline characteristics, it needs to be considered that ages and the ratio of medical history such as hypertension and diabetes mellitus in the EA group were disadvantageous than those in the control group.

Even though this study is meaningful in the point to be the first study attempted to quantitatively evaluate antispastic effect of EA on poststroke spasticity using T-reflex, but we could find neither electrophysiological nor clinical antispastic effect of EA. As a preliminary study, the limitations of this study include 29 small number of subjects, different number of allocated subjects in the study groups as EA group $(\mathrm{n}=19)$ and control group $(\mathrm{n}=10)$, and possibility of subject bias, that is overestimation of effect of treatment by the subject in the treatment group because assessors were blinded, but subjects and clinicians were not blinded in this study. Therefore, it is thought that large scale clinical trials for quantitative evaluation of effect of EA on poststroke spasticity will be needed.

\section{Acknowledgement}

This work was funded by Clinical Research Grant From Pusan National University Hospital 2015.

\section{Reference}

1. Statistics Korea. Cause of Death Statistics in 20 12. Available at: URL:http://kostat.go.kr/portal/k orea/kor_nw/3/ index.board?bmode=read\&aSeq= 308560. Accessed September 25, 2013.

2. Giroud M, Jacquin A, Béjot Y. The worldwide landscape of stroke in the 21st century. Lancet. 2014;383(9913):195-7. 
3. Wissel J, Manack A, Brainin M. Toward an epidemiology of poststroke spasticity. Neurology. 2013;80(3 Suppl 2):S13-9.

4. Lundström E, Smits A, Terént A, Borg J. Time-course and determinants of spasticity during the first six months following first-ever stroke. J Rehabil Med. 2010;42(4):296-301.

5. Lundström E, Smits A, Borg J, Terént A. Four-fold increase in direct costs of stroke survivors with spasticity compared with stroke survivors without spasticity: the first year after the event. Stroke. 2010;41(2):319-24.

6. Cho JH, Chung SH, Lee JS, Kim SS. MEMG Analysis on Antispastic Effect of Electroacupuncture and Transcutaneous Electrical Nerve Stimulation. J Oriental Rehab Med. 2006;16(2):131-143.

7. van der Salm A, Veltink PH, Ijzerman MJ, Groothuis-Oudshoorn KC, Nene AV, Hermens HJ. Comparison of electric stimulation methods for reduction of triceps surae spasticity in spinal cord injury. Arch Phys Med Rehabil. 2006; 87(2):222-8.

8. Moon SK, Whang YK, Park SU, Ko CN, Kim YS, Bae HS, et al. Antispastic effect of electroacupuncture and moxibustion in stroke patients. Am J Chin Med. 2003;31(3):467-74.

9. Wayne PM, Krebs DE, Macklin EA, Schnyer R, Kaptchuk TJ, Parker SW, et al. Acupuncture for upper-extremity rehabilitation in chronic stroke: a randomized sham-controlled study. Arch Phys Med Rehabil. 2005;86(12):2248-55.

10. You JH, Kim YS, Kang SK. Antispastic Effect of Electroacupuncture on Spasticity in Stroke Patients. J Kor Acu Mox Soc. 1999;16(2):1-12.

11. Kang SK, Kim YS. Effectiveness of Electroacupuncture on Spasticity in Stroke Patients. J Orint Med. 1997;2(1):25-32.

12. Decq P, Filipetti P, Lefaucheur JP. Evaluation of spasticity in adults. Operat tech Neurosurg. 2005;7:100-8.

13. Voerman GE, Gregoric M, Hermens HJ.
Neurophysiological methods for the assessment of spasticity: the Hoffmann reflex, the tendon reflex, and the stretch reflex. Disabil Rehabil. 2005;27(1-2):33-68.

14. Robert L. Quantified deep tendon reflex device, second generation. J Mech Med Biol. 2008;8(1): 75-85.

15. Min JH, Shin YI, Joa KL, Ko SH, Shin MJ, Chang $\mathrm{JH}$, et al. The Correlation between Modified Ashworth Scale and Biceps T-reflex and Inter-rater and Intra-rater Reliability of Biceps T-reflex. Ann Rehabil Med. 2012;36(4): 538-43.

16. Mutlu A, Livanelioglu A, Gunel MK. Reliability of Ashworth and Modified Ashworth scales in children with spastic cerebral palsy. BMC Musculoskelet Disord. 2008;9:44.

17. Levin MF, Hui-Chan CW. Relief of hemiparetic spasticity by TENS is associated with improvement in reflex and voluntary motor functions. Electroencephalogr Clin Neurophysiol. 1992;85(2): 131-42.

18. Goulet $\mathrm{C}$, Arsenault AB, Bourbonnais D, Laramée MT, Lepage Y. Effects of transcutaneous electrical nerve stimulation on H-reflex and spinal spasticity. Scand J Rehabil Med. 1996; 28(3):169-76.

19. Aydin G, Tomruk S, Keleş I, Demir SO, Orkun S. Transcutaneous electrical nerve stimulation versus baclofen in spasticity: clinical and electrophysiologic comparison. Am J Phys Med Rehabil. 2005;84(8):584-92.

20. Lee SW, Yun JM, Son JW, Kang BG, Park SM, Yun HJ, et al. The Effect of Electroacupuncture on Upper-Extremity Spasticity of Stroke Patients. Korean J Orient Int Med. 2007;28(3):492-501.

21. Kim EJ. The Effect of Quadriceps-Electrocutaneous Stimulation on the T-Reflex and the H-Reflex of the Soleus Muscle[Master's Thesis]. Seoul: E-wha Womans Univ.; 2000.

22. Péréon $\mathrm{Y}$, Nguyen The Tich S, Fournier E, Genet R, Guihéneuc P. Electrophysiological 
recording of deep tendon reflexes: normative data in children and in adults. Neurophysiol Clin. 2004;34(3-4):131-9.

23. Kim SW, Shin JB, You S, Kim HS, Nam JH, Song SH. Electrophysiological Changes after Botulinum Toxin Type A in Children with Cerebral Palsy. J Korean Acad Rehabil Med. 2010;34(2):179-84.

24. Stam J, van Crevel H. Reliability of the clinical and electromyographic examination of tendon reflexes. J Neurol. 1990;237(7):427-31.

25. Hefter H, Jost WH, Reissig A, Zakine B, Bakheit AM, Wissel J. Classification of posture in poststroke upper limb spasticity: a potential decision tool for botulinum toxin A treatment? Int J Rehabil Res. 2012;35(3):227-33.

26. Mukherjee M, McPeak LK, Redford JB, Sun C, Liu W. The effect of electro-acupuncture on spasticity of the wrist joint in chronic stroke survivors. Arch Phys Med Rehabil. 2007;88(2): 159-66.

27. Kim YW. Effects of Therapeutic Electrical Stimulation of Sensory Nerve Pathway on Spasticity in Patients with Brain Lesions [Master's Thesis]. Seoul:Yon-sei Univ.; 2001.

28. Levine MG, Knott M, Kabat H. Relaxation of spasticity by electrical stimulation of antagonist muscles. Arch Phys Med Rehabil. 1952;33(11): 668-73.

29. Filshie J, White A. Medical Acupuncture: A Western Scientific Approach. Seoul:KCA Press Company. 2001:160-1.

30. Chen XH, Han JS. All three types of opioid receptors in the spinal cord are important for 2/15 Hz electroacupuncture analgesia. Eur J Pharmacol. 1992;211(2):203-10.

31. Cho HY, In TS, Cho KH, Song $\mathrm{CH}$. A single trial of transcutaneous electrical nerve stimulation (TENS) improves spasticity and balance in patients with chronic stroke. Tohoku J Exp Med. 2013;229(3):187-93.
32. Tekeoğlu Y, Adak B, Göksoy T. Effect of transcutaneous electrical nerve stimulation (TENS) on Barthel Activities of Daily Living (ADL) index score following stroke. Clin Rehabil. 1998; 12(4):277-80.

33. Yan T, Hui-Chan CW. Transcutaneous electrical stimulation on acupuncture points improves muscle function in subjects after acute stroke: a randomized controlled trial. J Rehabil Med. 2009;41(5):312-6.

34. In TS, Cho HY, Lee SH, Lee JK, Song CH. The Long-Term Effects of High-Frequency Transcutaneous Electrical Nerve Stimulation (TENS) on the Lower Limb Spasticity and the Balance in the Chronic Stroke Patients. J Korea Acad Industr Coop Soc. 2011;12(4):1740-8.

35. Liu W, Mukherjee M, Sun C, Liu H, McPeak LK. Electroacupuncture may help motor recovery in chronic stroke survivors: a pilot study. J Rehabil Res Dev. 2008;45(4):587-95.

36. Wang $\mathrm{BH}$, Lin $\mathrm{CL}$, Li TM, Lin SD, Lin JG, Chou LW. Selection of acupoints for managing upper-extremity spasticity in chronic stroke patients. Clin Interv Aging. 2014;9:147-56.

37. Kim YS. Antispastic Effects of Electroacupuncture, TENS and NMES in Stroke Patient. J Kor Acu Mox Soc. 2000;17(2):209-20.

38. Chen XH, Guo SF, Chang CG, Han JS. Optimal conditions for eliciting maximal electroacupuncture analgesia with dense and disperse mode stimulation. Am J Acupunct. 1994;22(1):47-53.

39. Wang XB, Chen J, Li TJ, Tao J, Chen LD, He $\mathrm{J}$, et al. Effect of electroacupuncture in different frequencies on electromyography and ambulation in stroke patients with lower-extremity spasticity: a randomized controlled study. Zhongguo Zhen Jiu. 2011;31(7):580-4.

40. Han JS, Chen XH, Yuan Y, Yan SC. Transcutaneous electrical nerve stimulation for treatment of spinal spasticity. Chin Med J (Engl). 1994; 107(1):6-11. 